\title{
Saúde e segurança de trabalhadores da atenção primária durante o período de pandemia do covid-19: Rio Grande do Sul/Brasil
}

\author{
Health and safety of primary care workers during the pandemic period of COVID-19: \\ Rio Grande do Sul/Brazil
}

\author{
Suzane Beatriz Frantz Krug ${ }^{1}$ \\ Orcid: https://orcid.org/0000-0002-2820-019X \\ Bruna Rezende Martins ${ }^{3}$ \\ Orcid: https://orcid.org/0000-0001-5359-1228 \\ Ingre $\mathrm{Paz}^{5}$ \\ Orcid: https://orcid.org/0000-0002-2512-5278
}

\author{
Caroline Bertelli ${ }^{2}$ \\ Orcid: https://orcid.org/0000-0001-9961-4915 \\ Daiana Klein Weber Carissimi ${ }^{4}$ \\ Orcid: https://orcid.org/0000-0001-7438-4519 \\ Clauceane Venzke Zell ${ }^{6}$ \\ Orcid: https://orcid.org/0000-0002-9699-1843
}

\author{
Marcelo Carneiro ${ }^{7}$ \\ Orcid: https://orcid.org/0000-0003-3603-1987
}

\begin{abstract}
Resumo
Introdução: Profissionais da saúde estão entre a população exposta ao vírus da COVID-19 de forma direta, devido ao seu trabalho, na assistência e cuidado que dispensam às pessoas infectadas. Objetivos: analisar aspectos da saúde e segurança de trabalhadores da Atenção Primária em municípios do sul do Brasil, no contexto da pandemia da COVID-19. Materiais e métodos: pesquisa quantitativa e descritiva, realizada a partir de um estudo de base populacional. Os participantes foram profissionais de saúde da Atenção Primária de 13 municípios do interior do Rio Grande do Sul. A coleta de dados ocorreu em 2020, com questionário previamente estruturado composto por 34 perguntas fechadas. Avaliaram-se frequências absolutas e relativas. Resultados: a análise evidenciou que os profissionais da Atenção Primária deste estudo têm condições laborais adequadas, 60 (78,9\%) referiram não ter sofrido impacto com a pandemia, 19 (25\%) ficaram afastados devido a sintomas respiratórios, $74(97,4 \%)$ dos profissionais informaram ter modificado a forma e frequência de uso de EPIs, $51(67,1 \%)$ relataram que, durante a pandemia, houve investimento financeiro para compra de materiais e equipamentos por parte da sua instituição de trabalho e $64(84,2 \%)$ mencionaram a criação de novos fluxos e rotinas durante os atendimentos em saúde. Devido à situação pandêmica, alguns profissionais $(5,3 \%)$ precisaram afastar-se por contaminação, bem como, questões de saúde mental. Conclusões: Evidenciou-se a adesão dos trabalhadores às medidas de prevenção e o uso correto dos EPI durante a pandemia, bem como, a análise de diversos municípios possibilitou situações de trabalho distintas.

Palavras-chave: infecções por coronavírus; profissionais de saúde; condições de trabalho; segurança no trabalho; atenção primária à saúde.
\end{abstract}

\footnotetext{
${ }^{1}$ Universidade de Santa Cruz do Sul, Programa de Mestrado em Promoção da Saúde, área da saúde, Santa Cruz do Sul, Rio Grande do Sul, Brasil. E-mail: skrug@unisc.br

${ }^{2}$ Universidade de Santa Cruz do Sul, Programa de Mestrado em Promoção da Saúde, área da saúde, Santa Cruz do Sul, Rio Grande do Sul, Brasil. E-mail: caroline97bertelli@hotmail.com

${ }^{3}$ Universidade de Santa Cruz do Sul, Programa de Mestrado em Promoção da Saúde, área da saúde, Santa Cruz do Sul, Rio Grande do Sul, Brasil. E-mail: brezendem97@gmail.com

${ }^{4}$ Universidade de Santa Cruz do Sul, área da saúde, Santa Cruz do Sul, Rio Grande do Sul, Brasil. E-mail: dweber@unisc.br

${ }^{5}$ Universidade de Santa Cruz do Sul, Programa de Mestrado em Promoção da Saúde, área da saúde, Santa Cruz do Sul, Brasil. E-mail: ingrepaz@unisc.br

${ }^{6}$ Universidade de Santa Cruz do Sul, área da saúde, Santa Cruz do Sul, Rio Grande do Sul, Brasil. E-mail: clauceane@gmail.com

${ }^{7}$ Universidade de Santa Cruz do Sul, Programa de Mestrado em Promoção da Saúde, área da saúde, Santa Cruz do Sul, Rio Grande do Sul, Brasil. E-mail: marceloc@unisc.br
} 


\begin{abstract}
Introduction: Healthcare professionals are among the population directly exposed to the COVID19 virus, due to their work, in the assistance and care they provide to infected people. Objectives: to analyze health and safety aspects of Primary Care workers in municipalities of southern Brazil, in the context of the pandemic of the COVID-19. Materials and methods: quantitative and descriptive research, conducted from a population-based study. The participants were health professionals in Primary Care from 13 municipalities in the interior of Rio Grande do Sul. Data collection occurred in 2020, with a previously structured questionnaire consisting of 34 closed questions. Absolute and relative frequencies were evaluated. Results: The analysis showed that the Primary Care professionals in this study have adequate working conditions, 60 (78.9\%) reported not having been impacted by the pandemic, 19 (25\%) were absent due to respiratory symptoms, $74(97.4 \%)$ of professionals reported having changed the form and frequency of use of PPE, 51 (67.1\%) reported that, during the pandemic, there was financial investment for the purchase of materials and equipment by their work institution, and 64 (84.2\%) mentioned the creation of new flows and routines during health care. Due to the pandemic situation, some professionals $(5.3 \%)$ had to leave due to contamination, as well as mental health issues. Conclusions: The adherence of workers to preventive measures and the correct use of PPE during the pandemic were evidenced, as well as the analysis of several municipalities enabled distinct work situations.
\end{abstract}

Keywords: coronavirus infections; health personnel; working conditions; occupational health; primary health care.

\section{Introdução}

O relatório publicado pela Organização Mundial da Saúde, em maio de 2021, revela o quadro atual da pandemia ocasionada pela COVID-19, em que estão documentados mais de 166 milhões de casos confirmados e em torno de três milhões de mortes pela doença' ${ }^{1 \cdot}$ No Brasil, no mesmo período, o número de acometidos pelo vírus vem crescendo gradativamente e o país confirmou mais de 16 milhões de casos e 449 mil óbitos ${ }^{2}$.

O Brasil e muitos outros países têm concentrado suas ações de combate ao coronavírus em serviços hospitalares, ampliando o número de leitos, especialmente destinados a unidades de tratamento intensivo. Sem a intenção de retirar a importância desses feitos, alerta-se que na Atenção Primária à Saúde (APS), muito pode ser feito em situações de pandemia ${ }^{3}$. Para isso, é preciso estabelecer uma reorganização dos serviços da atenção básica no enfrentamento à pandemia $\mathrm{e}$ ainda, procurar manter a oferta das ações fundamentais realizadas nos territórios ${ }^{4}$.

As Estratégias de Saúde da Família (ESF), unidades integrantes da rede de APS caracterizam-se como a principal porta de entrada dos usuários no Sistema Único de Saúde (SUS) e destacam-se por suas ações de caráter multidisciplinar, baseadas na promoção, proteção e recuperação da saúde da população, agindo holisticamente e respeitando as demandas e individualidades de cada território ${ }^{5}$. Dessa forma, é necessário manter o contato e vínculo das pessoas com os profissionais, de forma a fortalecer o trabalho na rede de atenção primária ${ }^{3}$.

Os profissionais da saúde estão entre a população exposta ao vírus de forma direta, devido ao seu trabalho, na assistência e cuidado que dispensam às pessoas infectadas. A alta carga viral que estão suscetíveis a receber, bem como o estresse ao atender os pacientes, alguns em situações graves e as condições de trabalho, frequentemente inadequadas, são desafios a serem superados diariamente ${ }^{6,7}$. Assim, uma das maneiras de oferecer proteção e segurança aos profissionais muito conhecida e difundida, é o uso de Equipamentos de Proteção Individual (EPI), como máscaras, luvas, aventais, óculos, protetores faciais e através de protocolos de isolamento padrão, de contato e via aérea ${ }^{7}$.

Em uma situação de pandemia, o desgaste físico é comum entre os profissionais, no entanto, outro aspecto que precisa ser considerado diz respeito à saúde 
mental dos trabalhadores que atuam na linha de frente do COVID-19, nos mais diversos níveis de atenção. É necessário observar o cenário em que os profissionais estão inseridos, bem como considerar a carga de trabalho que é imposta aos mesmos, a qual impacta, ainda que de forma indireta, sua saúde mental. Dessa forma, é urgente a realização de medidas individuais, coletivas e institucionais que mitiguem os efeitos nefastos sobre os trabalhadores ${ }^{8,9}$.

A COVID-19 apresenta muitos impactos relacionados à saúde do trabalhador, por isso, a importância da prática de controle das infecções nos ambientes laborais, bem como, prevenção do contágio dos trabalhadores, considerando sua segurança física, condições de trabalho e estabilidade emocional e psíquica. Todos esses aspectos são primordiais para a continuação dos serviços e da qualidade de vida dos profissionais $^{10,11,12}$. Considera-se que $o$ enfrentamento da pandemia faz parte das funções essenciais da saúde pública, através de ações dirigidas à população ou para grupos com maior risco de contaminação, como por exemplo, os profissionais de saúde $^{13}$.

Ressalta-se que um fator determinante para a realização do presente estudo em municípios do Rio Grande do Sul/Brasil foi a opção por investigar trabalhadores da atenção primária em saúde, que vivenciam situações de trabalho com características e especificidades sociais, econômicas, políticas e culturais próprias e que podem ser determinantes para a organização do trabalho em situações pandêmicas, diferente da realidade de grandes centros urbanos.

Considerando o contexto de trabalho exercido na rede de atenção primária em saúde, com o intuito de conhecer as situações vivenciadas pelos profissionais de saúde no trabalho durante a pandemia e almejando servir de subsídio para futuras melhorias e adequações na saúde do trabalhador, este estudo tem por objetivo analisar aspectos da saúde e segurança de trabalhadores da atenção primária em municípios do sul do Brasil, no contexto da pandemia da COVID-19.

\section{Materiais e Métodos}

Trata-se de um estudo quantitativo, descritivo, recorte de um estudo de base populacional intitulado "Estudo de Soroprevalência de SARS-Cov-2 na Região do Vale do Rio Pardo (COVID-VRP)". Este artigo refere-se ao segundo momento de uma pesquisa que envolveu 14 municípios integrantes do Consórcio Intermunicipal de Serviços do Vale do Rio Pardo (CISVALE), localizado no interior do Rio Grande do Sul/Brasil, conforme apresentado na figura 1 . 
Figura 1 - Mapa da região de abrangência do CISVALE

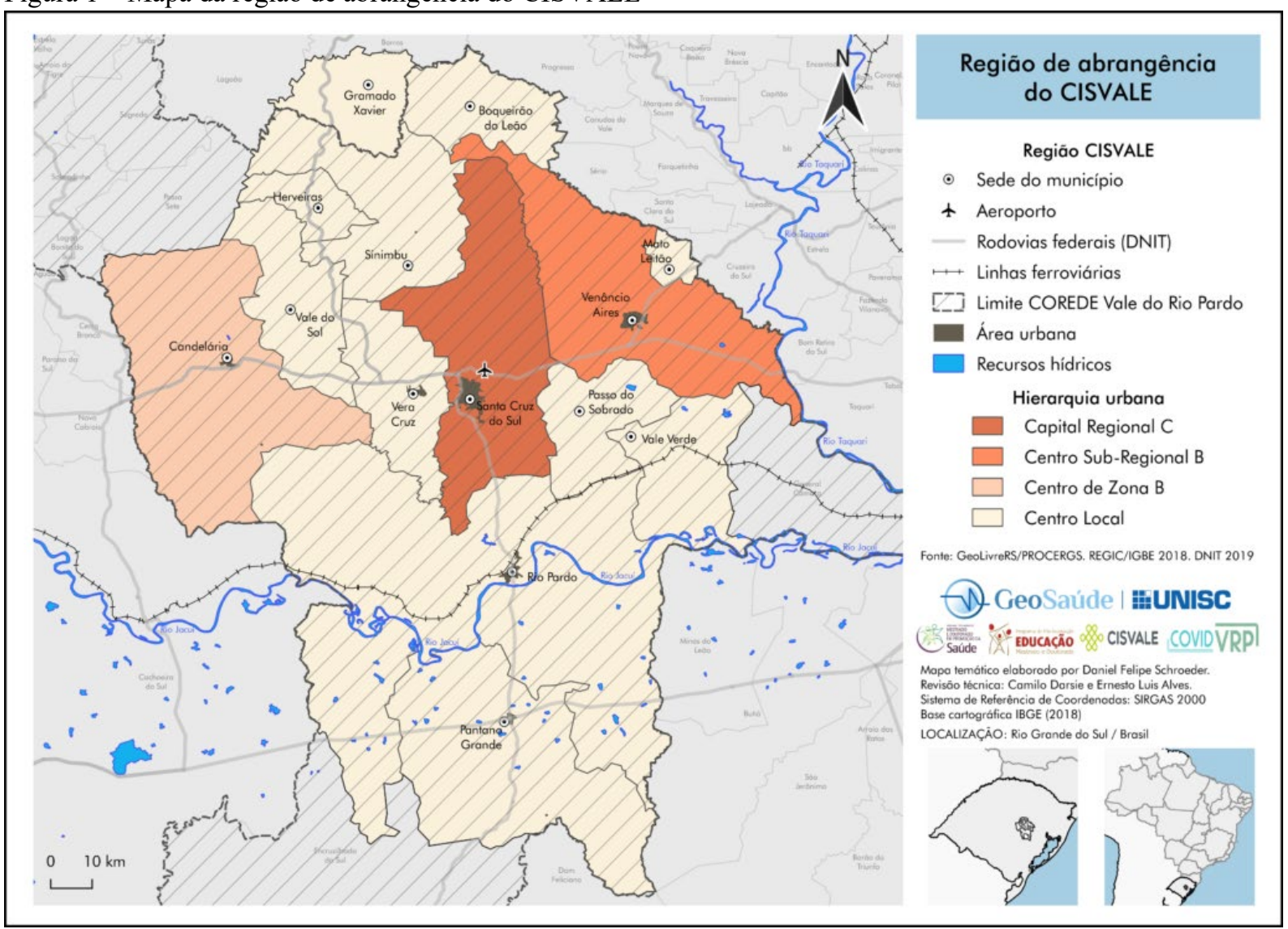

Fonte: SCHROEDER; DARSIE; ALVES, 202014

O primeiro momento do estudo de base populacional mensurou a soroprevalência de SARS-CoV-2 na população que faz parte do CISVALE. A coleta de dados ocorreu entre os meses de agosto a outubro de 2020, por meio da realização de testes rápidos e questionários junto à população. $\mathrm{O}$ segundo momento da pesquisa, a qual este artigo se refere, e cuja coleta de dados ocorreu em novembro de 2020, abrangeu profissionais de saúde da Atenção Primária de 13 dos 14 municípios do CISVALE.

Juntos, os municípios avaliados têm uma população aproximada de 228.788 habitantes, que possuem como característica comum extensas áreas rurais e predominantemente agrícolas, baseadas, principalmente, na produção de tabaco. A maioria das situações de vulnerabilidade em saúde dessa região são relativas ao trabalho na agricultura, processo de envelhecimento e agravos não transmissíveis. Quase em sua totalidade, os municípios citados possuem grande percentual de cobertura de Estratégia de Saúde da Família (ESF) e alguns contam com unidades hospitalares, que oferecem assistência de média complexidade, porém, muitos atendimentos de alta complexidade são encaminhados para centros de referência regionalizados, pactuados pela gestão.

Ao todo, 97 profissionais de saúde foram elegíveis para este estudo. Para tal, os critérios de inclusão foram: ser profissional da atenção primária à saúde e ter participado como coletador de teste rápido de sorologia da SARS - COV -2 e questionário junto à população de municípios do CISVALE.

Para a coleta dos dados utilizou-se um questionário previamente estruturado e testado, composto por 34 perguntas fechadas, foi criado e registrado na ferramenta Google Forms e enviado via email, com link de acesso aos profissionais. Durante o acesso, os participantes receberam um convite para participar do 
estudo, bem como orientações, esclarecimentos e o Termo de Consentimento Livre e Esclarecido (TCLE).

As informações coletadas foram digitadas no Excel e posteriormente, exportadas para um banco de dados, e, através do programa Jasp versão 013.1 foram realizadas análises estatísticas, avaliando frequências absolutas e relativas. O estudo foi aprovado pelo Comitê de Ética em Pesquisa da Universidade de Santa Cruz do Sul, sob o $n^{\circ}$ 4.278.695.

\section{Resultados}

Dos 97 profissionais de saúde elegíveis para o estudo, $76 \quad(78,3 \%)$ responderam ao questionário e integraram o presente estudo. A Tabela 1 apresenta os dados sociodemográficos dos participantes desta pesquisa.

Tabela 1 - Características sociodemográficas dos profissionais participantes.

\begin{tabular}{|c|c|c|}
\hline CARACTERÍSTICA & $N=76$ & $\%$ \\
\hline \multicolumn{3}{|l|}{ Sexo } \\
\hline Mulher & 74 & 97,3 \\
\hline Homem & 2 & 2,7 \\
\hline \multicolumn{3}{|l|}{ Cor da pele } \\
\hline Branca & 68 & 89,5 \\
\hline Parda & 6 & 7,9 \\
\hline Preta & 2 & 2,6 \\
\hline \multicolumn{3}{|l|}{ Estado civil } \\
\hline Casado (a) & 32 & 42,2 \\
\hline Solteiro (a) & 23 & 30,2 \\
\hline União Estável & 15 & 19,7 \\
\hline Divorciado (a) & 6 & 7,9 \\
\hline \multicolumn{3}{|l|}{ Faixa etária } \\
\hline De 31 - 40 anos & 28 & 37 \\
\hline De $41-50$ anos & 27 & 35,5 \\
\hline De $21-30$ anos & 12 & 15,8 \\
\hline De 51- 60 anos & 9 & 11,9 \\
\hline \multicolumn{3}{|l|}{ Religião } \\
\hline Católica & 48 & 63,2 \\
\hline Evangélica & 16 & 21,1 \\
\hline Espírita & 9 & 11,8 \\
\hline Sem religião & 2 & 2,6 \\
\hline Africana & 1 & 1,3 \\
\hline \multicolumn{3}{|l|}{ Escolaridade } \\
\hline Curso Técnico & 29 & 38,1 \\
\hline Pós-Graduação & 27 & 35,5 \\
\hline Curso Superior Completo & 12 & 15,9 \\
\hline Curso Superior Incompleto & 8 & 10,5 \\
\hline \multicolumn{3}{|l|}{ Renda familiar* } \\
\hline Entre 5 a 10 salários mínimos & 32 & 42.1 \\
\hline Entre 3 a 5 salários mínimos & 19 & 25 \\
\hline Entre 1 a 3 salários mínimos & 16 & 21,1 \\
\hline Entre 11 a 24 salários mínimos & 6 & 7,8 \\
\hline Entre meio e 1 salário-mínimo & 3 & 4 \\
\hline
\end{tabular}

*Considera-se o valor do salário-mínimo, de acordo com o piso nacional, R \$1.045.

Fonte: Dados do estudo, 2020. 
Este estudo foi composto predominantemente por trabalhadores de saúde do gênero feminino 74 (97,3\%), a maior parte dos entrevistados era da cor branca $68(89,5 \%)$, encontravam-se na faixa etária entre 31 a 40 anos, casados 32 (42,2\%), católicos $48(63,2 \%)$ e com formação técnica $29(38,1 \%)$.

A respeito dos aspectos familiares, $57(75 \%)$ dos profissionais afirmaram ter entre um e dois filhos. Ao analisar a amostra, destaca-se que $27(35,5 \%)$ dos entrevistados reside com seu companheiro e filhos, $16 \quad(21,1 \%)$ somente com companheiro, $13(17,1 \%)$ com familiares, aqui considerados os pais, tios, avós e/ou demais parentes, $12(15,8 \%)$ com filhos e 4 $(5,3 \%)$ moram sozinhos. Ademais, dentre o público com quem os profissionais da saúde residiam, $36(47,4 \%)$ da amostra eram crianças, $14(18,4 \%)$ idosos e $11(14,5 \%)$ apresentavam fatores de risco como hipertensão, diabetes, câncer, entre outros. A maior parte dos participantes, 32 $(42,1 \%)$, declarou ter uma renda individual entre $R \$ 5.641,60$ a $R \$ 11.279,13$, que, segundo a classificação da Associação Brasileira de Empresas de Pesquisa (ABEP) representa a classe $\mathrm{B}$, equivalente entre 5 e 10 salários mínimos e 3 (4\%) das pessoas declararam receber entre $\mathrm{R} \$ 719,81$ a $\mathrm{R} \$$ $1.748,58$, meio e um salário mínimo (classe E).

Quanto aos aspectos ocupacionais, conforme visualiza-se na tabela 2, 36 $(47,3 \%)$ dos respondentes eram técnicos de enfermagem, seguido por $28 \quad(36,8 \%)$ enfermeiros. Sobre o tempo de atuação na rede de saúde, 35 (46\%) dos participantes apresentavam mais de 10 anos de serviço e $5(6,6 \%)$ relataram estar há menos de um ano atuando na rede. Quando questionados a respeito de mais de um vínculo empregatício, $58(76,3 \%)$ possuem um vínculo, sendo que a maior parte $63(82,9 \%)$ trabalhava de 21 a 40 horas semanais, 63 $(82,9 \%)$ no turno manhã e $68(89,5 \%)$ na tarde. Verificou-se que $56(73,7 \%)$ atuavam nas unidades de atenção primária em saúde (Estratégias de Saúde da Família (ESF) e Unidades Básicas de Saúde (UBS)), localizadas na zona urbana $61(80,3 \%)$.

Tabela 2 - Características ocupacionais dos profissionais participantes.

\begin{tabular}{lcc}
\multicolumn{1}{c}{ DADOS OCUPACIONAIS } & N $=\mathbf{7 6}$ & $\mathbf{\%}$ \\
\hline Ocupação & 36 & 47,3 \\
Técnico de Enfermagem & 28 & 36,8 \\
Enfermeiro & 4 & 5,2 \\
Assistente Social & 3 & 3,9 \\
Cirurgião Dentista & 2 & 2,6 \\
Psicólogo & 1 & 1,3 \\
Terapeuta Ocupacional & 1,3 \\
Recepcionista & 1,3 \\
Médico & 1 & 46 \\
\hline Tempo de atuação profissional na rede de saúde & 29 \\
De 11 anos ou mais & 35 & 18,4 \\
De 1 - 5 anos & 22 & 6,6 \\
De 6 - 10 anos & 14 & \\
Menos de 1 ano & 5 & 76,3 \\
\hline Vínculos empregatícios & & 23,7 \\
Somente um vínculo empregatício & 58 & 82,9 \\
Mais de um vínculo empregatício & 18 & 10,5 \\
\hline Jornada de trabalho & & 5,3 \\
21-40 horas semanais & 63 & \\
41-60 horas semanais & 8 & \\
61-80 horas & 4 & \\
\hline
\end{tabular}




\begin{tabular}{lcc} 
Menos de 20 horas & 1 & 1,3 \\
\hline Local de trabalho & 56 & 73,7 \\
ESF/UBS & 10 & 13,1 \\
CAPS & 6 & 7,9 \\
Outros & 4 & 5,3 \\
Vigilância em Saúde & 47 & 61,9 \\
\hline Afastamento do trabalho durante a pandemia & 38,1 \\
Não & 29 & 25 \\
Sim & & 5,3 \\
\hline Razões do afastamento profissional & 19 & 4 \\
Sintomas respiratórios & 4 & 2,6 \\
Saúde mental & 3 & 1,3 \\
Fatores de risco para COVID-19 & 2 & \\
Devido à COVID-19 & 1 & \\
Acompanhamento familiar & & \\
\hline Fonte: Dados do estudo, 2020. & & \\
\hline
\end{tabular}

Fonte: Dados do estudo, 2020.

Quanto às questões relacionadas à saúde no trabalho, $47(61,9 \%)$ referiram não ter se afastado de suas atividades ocupacionais durante a pandemia, até o momento em que responderam à pesquisa. Contudo, destaca-se que, algumas das razões que culminaram no afastamento dos profissionais, foram sintomas respiratórios $(19-25 \%)$ e de sofrimento mental (4 5,3\%). Quando questionados acerca do impacto gerado pela pandemia na jornada de trabalho, $60(78,9 \%)$ referiram não ter sofrido alterações.

Ao abordar o tema segurança e proteção no trabalho (tabela 3), os participantes fizeram apontamentos acerca do uso de Equipamentos de Proteção Individual (EPI), sendo assim, 74 (97,4\%) dos profissionais informaram ter modificado a forma e frequência de uso dos mesmos após o início da pandemia, no sentido de aumentá-los para se protegerem. Ademais, segundo os respondentes 76 (100\%), o EPI mais utilizado foi a máscara $(100 \%)$, seguida por luvas $(76 \%)$, avental descartável, pijama cirúrgico e/ou Tyveck $(67,1)$, óculos $(56,6 \%)$ e face shield (48,7\%).

A maior parte dos trabalhadores 51 $(67,1 \%)$ relatou que durante a pandemia, houve investimento financeiro para compra de materiais e equipamentos por parte da sua instituição de trabalho. Além disso, 64 $(84,2 \%)$ dos profissionais relataram mudanças nos fluxos e rotinas nos locais de trabalho, bem como, 41 (53, 9\%) referiram ter recebido capacitações e/ou treinamentos sobre a COVID-19 (Tabela 3).

Tabela 3 - Dados de proteção e segurança no trabalho dos profissionais participantes na pandemia

\begin{tabular}{lcc}
\hline \multicolumn{1}{c}{ SEGURANÇA } & N $=\mathbf{7 6}$ & $\mathbf{\%}$ \\
\hline Modificações no uso de EPI & 74 & 97,4 \\
Sim & 2 & 2,6 \\
Não & 76 & \\
\hline EPI mais utilizados & 59 & 100 \\
Máscaras & 51 & 76 \\
Luvas & 43 & 67,1 \\
Avental descartável, pijama cirúrgico e/ou Tyveck & 37 & 56,6 \\
Face Shield & & 48,7 \\
Óculos & 51 & 67,1 \\
\hline Investimentos para a saúde & 25 & 32,9 \\
Sim & & \\
Não &
\end{tabular}




\begin{tabular}{lcc}
\hline Mudanças nos fluxos e rotinas no local de trabalho & 64 & 84,2 \\
Sim & 12 & 15,8 \\
Não & & \\
\hline Capacitações e/ou treinamentos sobre COVID-19 & 41 & 53,9 \\
Sim & 35 & 46,1 \\
Não
\end{tabular}

Fonte: Dados do estudo, 2020.

\section{Discussão}

Ao analisar o perfil sociodemográfico dos sujeitos deste estudo, observou-se predominância do sexo feminino entre os profissionais de saúde da APS, o que vai ao encontro de um estudo realizado em Estratégias de Saúde da Família da Região Sul, onde 95,1\% dos sujeitos eram mulheres, confirmando-se a tendência de feminização da força de trabalho na atenção básica ${ }^{15,16}$. Tal situação pode ser justificada pelo fato de as mulheres representarem a maior força de trabalho, principalmente em atividades relacionadas à saúde, se comparadas com o sexo masculino $^{17}$.

Em relação à cor da pele, a maior parte dos participantes desta pesquisa declara-se de cor branca, dado corroborado também em uma pesquisa da Fiocruz $(2020)^{18}$ que avaliou mais de 25 mil profissionais de saúde brasileiros, onde $57,7 \%$ também declararam-se brancos. No que se refere à situação conjugal, um estudo realizado com 34 equipes de saúde da família do estado da Paraíba identificou que a maioria dos profissionais de enfermagem vinculados à ESF eram casados ${ }^{19}$, resultados semelhantes aos dados da presente pesquisa.

Quanto à faixa etária dos profissionais, predominantemente de 30 a 40 anos, percebe-se que a mesma está em consonância com a de outros estudos, os quais discutiram que a maioria dos profissionais atuantes na APS são adultos jovens ${ }^{19,20}$. Quanto à escolaridade dos participantes desta pesquisa, a maioria apresentava nível técnico, seguidos por profissionais que possuíam pós-graduação. A grande quantidade de trabalhadores pósgraduados pode inferir uma tendência de busca de melhores qualificações, o que também é corroborado por outras pesquisas $^{15,21}$.

É possível destacar que a posição socioeconômica influencia diretamente no cotidiano dos indivíduos, isto é, pode aumentar as vulnerabilidades, bem como, oferecer possibilidades de vida mais saudáveis ${ }^{22}$. Nessa direção, nesta pesquisa evidenciou-se que a maior parte dos trabalhadores participantes possuía um alto padrão socioeconômico, isto é, recebia de cinco a dez salários mínimos e estaria inserido na Classe $\mathrm{B}$, de acordo com a ABEP. Essa realidade se difere da vivenciada pela população geral dos 13 municípios avaliados, os quais apresentamse inseridos na Classe D, segundo dados disponibilizados pela Fundação Getúlio Vargas (2021) e ABEP. Compreende-se que a condição econômica é determinante para mudanças nas condições de vida, isso é, padrões econômicos elevados possibilitam mais atividades de lazer, investimentos em práticas de exercícios, acompanhamento médico e capacitações profissionais $^{23}$.

Quanto aos moradores que residiam com os participantes desta pesquisa, $14,5 \%$ dos familiares apresentavam fatores de risco como hipertensão, diabetes, câncer, entre outros. Sobre isso, autores refletem que sentimentos como ansiedade, medo e estresse são experienciados pelos profissionais expostos ao vírus em seu local de trabalho, devido ao receio de infectar seus familiares, especialmente os que sofrem com comorbidades e idosos ${ }^{7}$.

No que concerne às características ocupacionais dos trabalhadores desta pesquisa, destaca-se que a maioria dos profissionais são enfermeiros, o que vai ao 
encontro de uma pesquisa realizada em Santa Rosa, no Rio Grande do Sul, em que $47,4 \%$ pertenciam a essa categoria profissional ${ }^{24}$. A maior parte dos profissionais possuíam mais de dez anos de serviços na APS, o que pode representar movimentos de estagnação e consolidação das atividades desenvolvidas ${ }^{16}$. Autores inferem que a permanência dos profissionais por maiores períodos proporciona contatos efetivos com a comunidade, bem como facilita o reconhecimento das demandas em saúde e a continuidade de programas de saúde nos territórios adscritos ${ }^{24}$.

Ainda quanto à temática, foi possível verificar que o cumprimento de uma carga horária integral favorece $o$ desempenho das atividades, bem como a formação de vínculos entre os membros da equipe e com a comunidade atendida, fatores que refletem diretamente na qualidade do atendimento prestado ${ }^{15}$. Outra característica observada neste estudo foi o elevado número de trabalhadores dedicando-se exclusivamente às atividades na atenção primária à saúde, cumprindo carga horária de 40 horas semanais, semelhante ao verificado em João Pessoa $(\mathrm{PA})^{25}$.

Neste estudo, $25 \%$ dos profissionais precisaram afastar-se de suas atividades laborais na rede de saúde por apresentarem sintomas respiratórios, devido à suspeita de COVID-19. Os trabalhadores, além de vivenciarem o afastamento dos colegas de profissão, ainda acabam sobrecarregados pelas atividades nas unidades de saúde ${ }^{26}$. O desgaste físico e mental dos profissionais também pode estar vinculado à possibilidade de falta de materiais, medicamentos, bem como, o fato de precisarem lidar com desfechos críticos/trágicos ${ }^{27}$.

Conforme supracitado, acerca das causas geradoras de afastamentos dos profissionais da Atenção Primária à Saúde (APS) durante a pandemia, este estudo também destaca o sofrimento mental, relatado por $4 \%$ dos participantes, como motivo para o absenteísmo. Sabe-se que os impactos na saúde mental decorrentes de níveis de estresse elevados durante períodos epidêmicos tendem a comprometer a atenção, bem como a capacidade de decisão dos trabalhadores, o que afeta não somente a luta contra o coronavírus, mas pode apresentar um efeito duradouro no bemestar dos profissionais ${ }^{28}$. Dessa forma, incentivar a construção de redes de apoio entre colegas, família ou comunidade, ainda que por meio de ferramentas digitais, configura-se como um recurso importante para uma boa saúde emocional ${ }^{29}$. O acolhimento e o estímulo a espaços de diálogo são intervenções fundamentais que tendem a fortalecer vínculos entre os profissionais e melhorar o desenvolvimento de seus processos de trabalho ${ }^{13}$.

Diferentemente do que foi visualizado na pesquisa da Fiocruz $(2021)^{18}$, citada anteriormente, onde os profissionais admitiram mudanças nas suas jornadas de trabalho em função da pandemia, os participantes desta pesquisa relataram que não houve modificações com sua carga de trabalho e com isso conseguiram permanecer com seus horários cotidianos.

No que diz respeito ao item de proteção e segurança, os autores inferem que adequações nos serviços de saúde da Atenção Primária devem ser estabelecidas, no sentido de proporcionar EPI a todos os profissionais de saúde ${ }^{30}$. Nessa direção, acerca do uso desses equipamentos, os participantes deste estudo, de maneira geral, ao responderem as perguntas do questionário, relatam ter aumentado a frequência do uso dos mesmos com a pandemia, bem como, estarem utilizandoos de forma correta e completa, a fim evitar o contágio pelas várias vias de infecção. A realidade vivenciada pelos sujeitos desta pesquisa difere de outros estudos, os quais apresentam um contexto diferente em outros municípios brasileiros em 2020, em que a disponibilidade de EPI, após três meses do início da pandemia, ainda era escassa $^{31}$. Ademais, 24\% dos profissionais 
assinalaram a disponibilidade de um conjunto de EPI - proteção facial, ocular, luvas e avental nas UBS.

Ainda voltado aos aspectos de segurança, os locais de atendimento nas unidades de saúde precisam ser diferenciados para cada atividade realizada e a higienização deve ser constante, servindo estas de medidas para mitigação da contaminação dos profissionais. Importante ressaltar que caso não sejam observadas as condutas necessárias para diminuir o risco de infecção nos profissionais que compõem as equipes de saúde, o funcionamento das mesmas pode apresentar mais risco do que auxílio na pandemia $^{32}$.

A sobrecarga de trabalho e a precariedade das instalações de saúde servem como interferências na adesão a protocolos e medidas de biossegurança. Dessa forma, cabe à gestão do trabalho dispor de meios dignos e seguros aos profissionais, a fim de garantir sua saúde, particularmente num contexto pandêmico, em que o risco de contaminação pelo coronavírus é alto $(26,33)$. A presente pesquisa demonstra que mais da metade dos profissionais entrevistados percebe investimentos financeiros por parte da instituição/gestão, diferentemente de outras localidades brasileiras, que conforme destacado por Bousquat et al. (2020) enfrentam cada vez mais dificuldades com a falta de verbas, o que ocasiona o sucateamento das unidades de saúde, bem como a falta de materiais e de mão de obra $^{31}$.

A maioria dos participantes desta pesquisa confirmou a instauração de novos fluxos, bem como alterações nas rotinas e durante os atendimentos nos seus locais de trabalho. Corroborando essa informação, a Associação Brasileira de Enfermagem (2020) destacou que diversas modificações precisaram ser instituídas para que os trabalhadores pudessem adequar-se durante a pandemia. Sendo assim, este atual contexto de saúde obrigou os profissionais a se reinventarem, restabelecerem e criarem novos planos de cuidados e, que fossem voltados, prioritariamente, à gestão e à atenção à saúde de casos respiratórios ${ }^{33}$.

As capacitações ou treinamentos recebidos sobre a COVID-19 foram uma realidade apresentada pelos participantes desta pesquisa, e acerca disso, Medina e colaboradores (2021) inferem que para o enfrentamento da pandemia a qual estamos vivenciando, é necessária a constante formação e educação permanente dos profissionais de saúde, fortalecendo assim, a segurança desses trabalhadores na assistência à saúde, bem como, proporciona uma maior qualidade no atendimento aos usuários $^{3}$. As novas estratégias em saúde devem ser ágeis e amplas, a fim de que sejam remodeladas para o contexto em que estamos inseridos, em que a maior parte das formas de comunicação ocorre à distância, de modo remoto ${ }^{31}$.

A Atenção Primária à Saúde é notada também por ser uma enorme potência contra a pandemia a qual estamos enfrentando e isso se dá devido à diversidade de profissionais, ao tamanho das redes de saúde, bem como suas funções referentes à vigilância em saúde e ao cuidado integral, igualitário e universal ${ }^{31}$.

\section{Conclusão}

Os profissionais da atenção primária em saúde deste estudo contam com uma rede de apoio fortalecida e organizada em seus locais de trabalho. Um número pequeno de participantes precisou afastarse de suas atividades laborais durante a pandemia, embora os motivos mais citados sejam preocupantes: sintomas respiratórios e sofrimento mental.

O estudo mostrou que o trabalho na pandemia na APS destacou a importante e evidente adesão dos trabalhadores da saúde às medidas de prevenção, de forma geral e muito intensamente ao uso correto e permanente dos EPI, medida essa sempre tão destacada e orientada em qualquer situação e ambiente de trabalho. Ademais, o 
estudo em vários serviços de saúde da Atenção Primária em Saúde de diversos municípios do interior do RS, cada qual com sua realidade de trabalho, foi um diferencial da pesquisa, já que a localização geográfica regional mostrou que as situações de trabalho na pandemia da COVID-19 não são distintas entre eles.

Os resultados trazidos pela pesquisa apontam para a necessidade de mais estudos que demonstrem os impactos ocasionados pela COVID-19 no trabalho na Atenção Primária à Saúde, a fim de que se tenha um maior conhecimento científico para lidar com outros eventos críticos como o que estamos vivenciando, com o intuito de manter e/ou criar estratégias eficazes no campo da saúde pública e coletiva.

\section{Referências Bibliográficas}

1. Organização Pan-Americana de Saúde. Folha informativa COVID-19 - Escritório da OPAS e da OMS no Brasil; 2021. Disponível em: https://covid19.who.int/ Acessado em 13 de maio de 2021.

2. Brasil. Ministério da Saúde. Painel coronavírus. Atualizada em 23 de maio de 2021; 2021. Disponível em: https://covid.saude.gov.br/ Acessado em 23 de maio de 2021.

3. Medina MG, Giovanella L, Bousquat A, Mendonça MHMD, Aquino R. Atenção primária à saúde em tempos de COVID-19: o que fazer? Cadernos de Saúde Pública. 2020; 36 (8): 1-5. doi: http://dx.doi.org/10.1590/0102-311X00149720

4. Engstrom E, Melo E, Giovanella L, Mendes A, Grabois V, De Mendonça MHM. Recomendações para a organização da Atenção Primária à Saúde no SUS no enfrentamento da COVID-19. [Internet]. 2020. [citado em 04 mar 2021]. Disponível em: https://portal.fiocruz.br/documento/recomendacoes-para-organizacao-da-aps-nosus-no-enfrentamento-da-COVID-19 Acessado em: 24 de maio de 2021.

5. Rezende BM, Bertelli C, Almeida KO, Zell CV, Franz EBT, De Oliveira L, et al. Construção do perfil sociodemográfico e clínico de indivíduos de uma microárea pertencente a uma estratégia de saúde da família: ações do pet-graduasus. Revista Interdisciplinar de Promoção da Saúde. 2018; 1 (4): 267-73. doi: http://dx.doi.org/10.17058/rips.v1i4.13097

6. Ribeiro AP, Oliveira GL, Silva LS, Souza ERD. Saúde e segurança de profissionais de saúde no atendimento a pacientes no contexto da pandemia de COVID-19: revisão de literatura. Revista Brasileira de Saúde Ocupacional. 2020; 45 (25): 1-12. doi: https://doi.org/10.1590/2317-6369000013920

7. Teixeira CFDS, Soares CM, Souza EA, Lisboa ES, Pinto ICDM, Andrade LRD, et al. A saúde dos profissionais de saúde no enfrentamento da pandemia de COVID-19. Ciência \& Saúde Coletiva. 2020; 25 (9): 3465-74. doi: https://doi.org/10.1590/141381232020259.19562020

8. Rego S, Palácios M. Saúde mental dos trabalhadores de saúde em tempos de coronavírus. Informe ENSP, p.1, 2020. [Internet]. 2020. [citado em 27 mar 2021]. Disponível em: https://www.arca.fiocruz.br/handle/icict/40659. Acessado em: 16 de março 2021. 
9. Miranda FMDA, De Lima Santana L, Pizzolato AC, Sarquis, LMM. Condições de trabalho e o impacto na saúde dos profissionais de enfermagem frente a COVID-19. 2020; 25 (e72702): 1-8. doi: http://dx.doi.org/10.5380/ce.v25i0.72702

10. Koh D. Occupational risks for COVID-19 infection. Occupational medicine. 2020; 70 (1): 3-5. doi: https://doi.org/10.1093/occmed/kqaa036

11. Huh S. How to train health personnel to protect themselves from SARS-CoV-2 (novel coronavirus) infection when caring for a patient or suspected case. Journal of educational evaluation for health professions. 2020; 17(10): 1-6. DOI: 10.3352/jeehp.2020.17.10.

12. Bahl P, Doolan C, De Silva C, Chughtai AA, Bourouiba L, MacIntyre CR. Airborne or droplet precautions for health workers treating COVID-19?. The Journal of infectious diseases. 2020; 10: 1-8. DOI: 10.1093/infdis/jiaa189.

13. Martins RU, De Araújo VA, Da Silva RN, Tavares BLL, De Oliveira Barcelos LS, Da Silva RA. Saúde Mental dos Profissionais da Atenção Básica em Tempos de Pandemia. Cadernos ESP-Revista Científica da Escola de Saúde Pública do Ceará. 2020; 14 (1): 133-37. https://doi.org/10.33233/eb.v19i4.4381;

14. Schroeder DF, Darsie C, Alves EL. R, cartógrafos. Região de abrangência do CISVALE [mapa]. Santa Cruz do Sul: GeoSaúde; 2020.

15. Moreira IJB, Horta JA, Duro LN, Borges DT, Cristofari AB, Chaves J, et al. Perfil sociodemográfico, ocupacional e avaliação das condições de saúde mental dos trabalhadores da Estratégia Saúde da Família em um município do Rio Grande do Sul, RS. Revista brasileira de medicina de família e comunidade. 2016; 11 (38): 1-12. doi: https://doi.org/10.5712/rbmfc11(38)967.

16. Marsiglia RMG. Perfil dos trabalhadores da atenção básica em saúde no município de São Paulo: região norte e central da cidade. Saúde e Sociedade. 2011; 20 (4): 900-11. doi: https://doi.org/10.1590/S0104-12902011000400008

17. Bertelli C, Martins BR, Krug SBF, Petry AR, Fagundes PDS. Acidentes de trabalho com material biológico: perfil sociodemográfico e ocupacional dos trabalhadores afetados. Revista Brasileira de Medicina do Trabalho. 2020; 18 (4): 415-424. doi: . http://dx.doi.org/10.47626/1679-4435-2020-534

18. Fiocruz. COVID-19: Estudo avalia condições de trabalho na Saúde. [Internet]. 2020. [citado em 27 mar 2021]. Disponível em: https://agencia.fiocruz.br/COVID-19-estudoavalia-condicoes-de-trabalho-na-saude Acessado em 10 de abril de 2021.

19. Oliveira MM, Pedraza DF. Contexto de trabalho e satisfação profissional de enfermeiros que atuam na Estratégia Saúde da Família. Saúde em Debate. 2019; 43 (122): 765-79. doi: https://doi.org/10.1590/0103-1104201912209

20. Meira KC, Myrrha LJD, De Jesus JC, De Oliveira JSA, Silva PDS. O perfil e a sobrecarga na jornada de trabalho de profissionais da linha de frente ao combate à pandemia da COVID-19. Observatório do Nordeste para Análise Sociodemográfica da 
COVID-19 da Universidade Federal do Rio Grande do Norte [Internet]. 2020. [citado em 27 mar 2021]. Disponível em: https://demografiaufrn.net/2020/05/13/sobrecargalinha-frente/ Acessado em: 24 de maio de 2021.

21. De Melo Costa S, Prado MCM, Andrade TN, Araújo EPP, Silva Junior W, Gomes Filho ZC, et al. Perfil do profissional de nível superior nas equipes da Estratégia Saúde da Família em Montes Claros, Minas Gerais, Brasil. Revista Brasileira de Medicina de Família e Comunidade. 2013; 8 (27), p. 90-96. doi: https://doi.org/10.5712/rbmfc8(27)530

22. Faleiro JC, Giatti L, Barreto SM, Camelo LDV, Griep RH, Guimarães J, et al. Posição socioeconômica no curso de vida e comportamentos de risco relacionados à saúde: ELSA-Brasil. Cadernos de Saúde Pública. 2017; 33 (3): 1-16. doi: https://doi.org/10.1590/0102-311X00017916

23. Knob Pintom B, Bisognin E, Da Rosa F, Maron LC. Perfil de trabalhadores da saúde da Atenção Primária de Santa Rosa/RS. Revista Saúde. 2016; 9 (2): 20-31.

24. Zanetti TG, Van der Sand ICP, Girardon-Perlini NMO, Kopf ÁW, De Abreu PB. Perfil socioprofissional e formação de profissionais de equipes de saúde da família: um estudo de caso. Ciência, Cuidado e Saúde. 2010; 9 (3): 448-55. doi: https://doi.org/10.4025/cienccuidsaude.v9i3.7664

25. De Brito GEG, Mendes ADCG, Dos Santos Neto PM, De Farias DN. Perfil dos trabalhadores da Estratégia Saúde da Família de uma capital do nordeste do Brasil. Revista de APS. 2016; 19 (3): 434-445.

26. Vedovato TG, Andrade CB, Santos DL, Bitencourt SM, Almeida LPD, Sampaio JFDS. Trabalhadores (as) da saúde e a COVID-19: condições de trabalho à deriva? Revista Brasileira de Saúde Ocupacional. 2020; 46 (e1): 1-15. doi: https://doi.org/10.1590/2317-6369000028520

27. Dantas ESO. Saúde mental dos profissionais de saúde no Brasil no contexto da pandemia por COVID-19. Interface-Comunicação, Saúde, Educação. 2021; 25 (Supl.1): 1-9. doi: https://doi.org/10.1590/Interface.200203

28. Kang L, Li Y, Hu S, Chen M, Yang C, Yang BX, et al. The mental health of medical workers in Wuhan, China dealing with the 2019 novel coronavirus. The Lancet Psychiatry. 2020; 7 (3): 1-9. doi: https://doi.org/10.1016/S2215-0366(20)30047-X

29. Brasil. Recomendações e orientações em saúde mental e atenção psicossocial na COVID-19. [Internet]. 2020 [citado em 09 de mar 2021]. Disponível em: https://www.fiocruzbrasilia.fiocruz.br/wpcontent/uploads/2020/10/livro_saude_mental_covid19_Fiocruz.pdf Acessado em: 15 de maio de 2021.

30. Soares CSA, Da Fonseca CLR. Atenção Primária à saúde em tempos de pandemia. Journal of Management \& Primary Health Care. 2020; 12 (22): 1-11. doi: https://doi.org/10.14295/jmphc.v12.998 
31. Lima JG, Giovanella L, Bousquat A, Mota PHS, Silva Júnior CL, Nedel F, et al. Desafios da Atenção Básica no enfrentamento da pandemia da COVID-19 no SUS. Relatório de Pesquisa. USP, Fiocruz, UFBA, UFPEL, OPAS Brasil. Rede de Pesquisa em Atenção Primária à Saúde da Abrasco. [Internet]. 2020. Disponível em: https://redeaps.org.br/wpcontent/uploads/2020/08/RelatorioDesafiosABCovid19SUS.pdf. Acessado em 14 de abril de 2021.

32. Tasca R, Massuda A. Estratégias para reorganização da Rede de Atenção à Saúde em resposta à Pandemia COVID-19: a experiência do Sistema de Saúde Italiano na região de Lazio. APS em Revista. 2020; 2 (1): 20-7. doi: https://doi.org/10.14295/aps.v2i1.65

33. Jackson Filho JM, Assunção AA, Algranti E, Garcia EG, Saito CA, Maeno M. A saúde do trabalhador e o enfrentamento da COVID-19. Rev bras saúde ocup. 2020; 45 (14): 1-3. doi: https://doi.org/10.1590/2317-6369ED0000120

\section{Como citar este artigo:}

Krug SBF, Bertelli C, Martins BR, Carissimi DKW, Paz I, Zell CV. Carneiro M. Saúde e segurança de trabalhadores da Atenção Primária durante o período de pandemia do COVID-19: Rio Grande do Sul/Brasil. Rev. Aten, Saúde. 2021; 19(70): 221-234. 